\title{
Designing issues of the geodetic vertical reference network of Lithuania
}

\author{
Boleslovas Krikštaponis, Eimuntas Kazimieras Paršeliūnas, Povilas Viskontas, \\ Algirdas Neseckas, Evelina Zigmantienè, Ričardas Kolosovskis, Arvydas Musteikis, \\ Eglè Tumelienè, Rosita Birvydienè, Petras Petroškevičius \\ Vilnius Gediminas Technical University, Sauletekio av. 11, 10223 Vilnius, Lithuania
}

\begin{abstract}
Activities of Lithuanian National Geodetic Vertical Reference Network (NGVRN) establishment are going on since 1998. The goal of NGVRN establishment is a creation of new Lithuanian height reference suitable for present period practical and scientific needs. The Lithuanian Geodetic Vertical First Order Network consists of five polygons. Perimeter of the network is ca. $1900 \mathrm{~km}$. Its development was finished in 2006. Connections of the first order vertical network with the vertical networks of neighbouring countries were established. All this creates good preconditions for determination of relations between height systems and for introduction of a new Lithuanian geodetic vertical (height) system. But this network is not dense enough to transfer the geodetic vertical system to the all territory of Lithuania and to improve the geoid model. It is necessary to dense available First Order Geodetic Vertical Network by developing the Second Order Geodetic Vertical Network.

The territory of Lithuania is divided into five regions. Borders of the regions are First Order Network levelling lines and lines connecting Lithuanian national Vertical Network to the corresponding networks of the neighbouring countries. The regions are called: South, East, North, West and Centre. The design of the Second Order Geodetic Vertical Network is presented. The necessary density, accuracy of the geopotential numbers and ellipsoidal heights are discussed. Some results of the geodetic measurements are presented too.
\end{abstract}

Keywords: Geodetic Vertical Network; levelling; geopotential number; ellipsoidal height.

\section{Introduction}

Activities of Lithuanian National Geodetic Vertical Reference Network (NGVRN) establishment are going on since 1998 [1-8]. The goal of NGVRN establishment is a creation of new Lithuanian height reference suitable for present period practical and scientific needs. The Lithuanian Geodetic Vertical First Order Network consists of five polygons. Perimeter of the network is ca. $1900 \mathrm{~km}$. Its development was finished in 2006. Connections of the first order vertical network with the vertical networks of neighbouring countries were established (one - with Polish vertical network, three - with Latvian vertical network) [9-11]. All this creates good preconditions for determination of relations between height systems and for introduction of a new Lithuanian geodetic vertical (height) system. But this network is not dense enough to transfer the geodetic vertical system to the all territory of Lithuania and to improve the geoid model. It is necessary to dense available First Order Geodetic Vertical Network by developing the Second Order Geodetic Vertical Network. The project of the Second order network was prepared in 2005-2006 [12], therefore its realisation was stopped by economic crisis. Nowadays the works to develop the Second Order Geodetic Vertical Network of Lithuania are going on.

\section{Design of the Second Order Geodetic Vertical Network}

The territory of Lithuania (65.3 th.sq.km) is divided in to five regions. Borders of the regions are First Order Network levelling lines and lines connecting Lithuanian national Vertical Network to the corresponding networks of the neighbouring countries. The regions are called: South, East, North, West and Centre. Fig. 1 represents a division scheme of Lithuania into regions.

Corresponding author: Boleslovas Krikštaponis. E-mail address: boleslovas.krikstaponis@vgtu.lt

http://dx.doi.org/10.3846/enviro.2014.223

(C) 2014 The Authors. Published by VGTU Press. This is an open-access article distributed under the terms of the Creative Commons Attribution License, which permits unrestricted use, distribution, and reproduction in any medium, provided the original author and source are credited. 


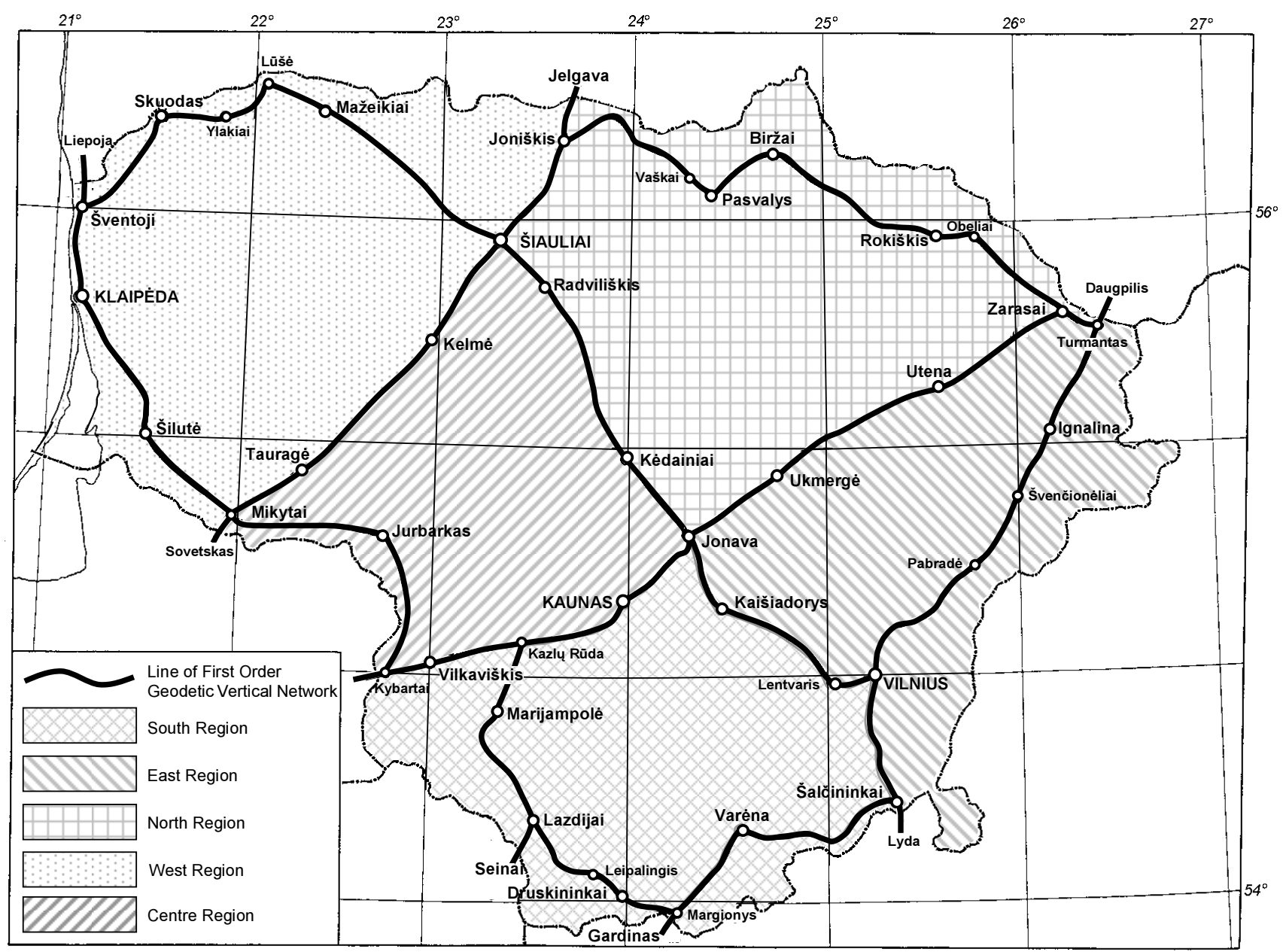

Fig. 1. Scheme of First Order Geodetic Vertical Network of Lithuania and division of the country's territory to the regions

Lines of Second Order Geodetic Vertical Network are arranged in such way that any point inside or outside the polygon will be in less than $30 \mathrm{~km}$ away from benchmarks of First and Second Order Network. Were possible levelling lines were projected through gravimetric points of first class, existing National levelling benchmarks and National GPS Network points (which are mounted in appropriate way).

The project of Second Order Network of each region was created individually, based on the benchmarks of First Order Network which are in territory of this region. Existing road network, relief and specific conditions of locality was taken into account. Former levelling lines were used for Network of Second Order project (Fig. 2).

Position of planned levelling lines are determined, length of each line and information about the existing levelling benchmarks was collected. Projected lines are marked by codes - numerical fractions (First Order Polygon number in numerator and Second Order line number in denominator). The project provides main necessary information to plan the reconnaissance of projected lines in the countryside and to locate existing old levelling benchmarks. Connections of the second order vertical network with the vertical networks of neighbouring countries (Latvia, Poland, Belarus and Russia) are foreseen.

About 2250 points, 1200 of them newly built should be included into the Second Order Network, following regulations, that the mean distance between benchmarks in the lines of Vertical Network should be less than $1.5 \mathrm{~km}$. 


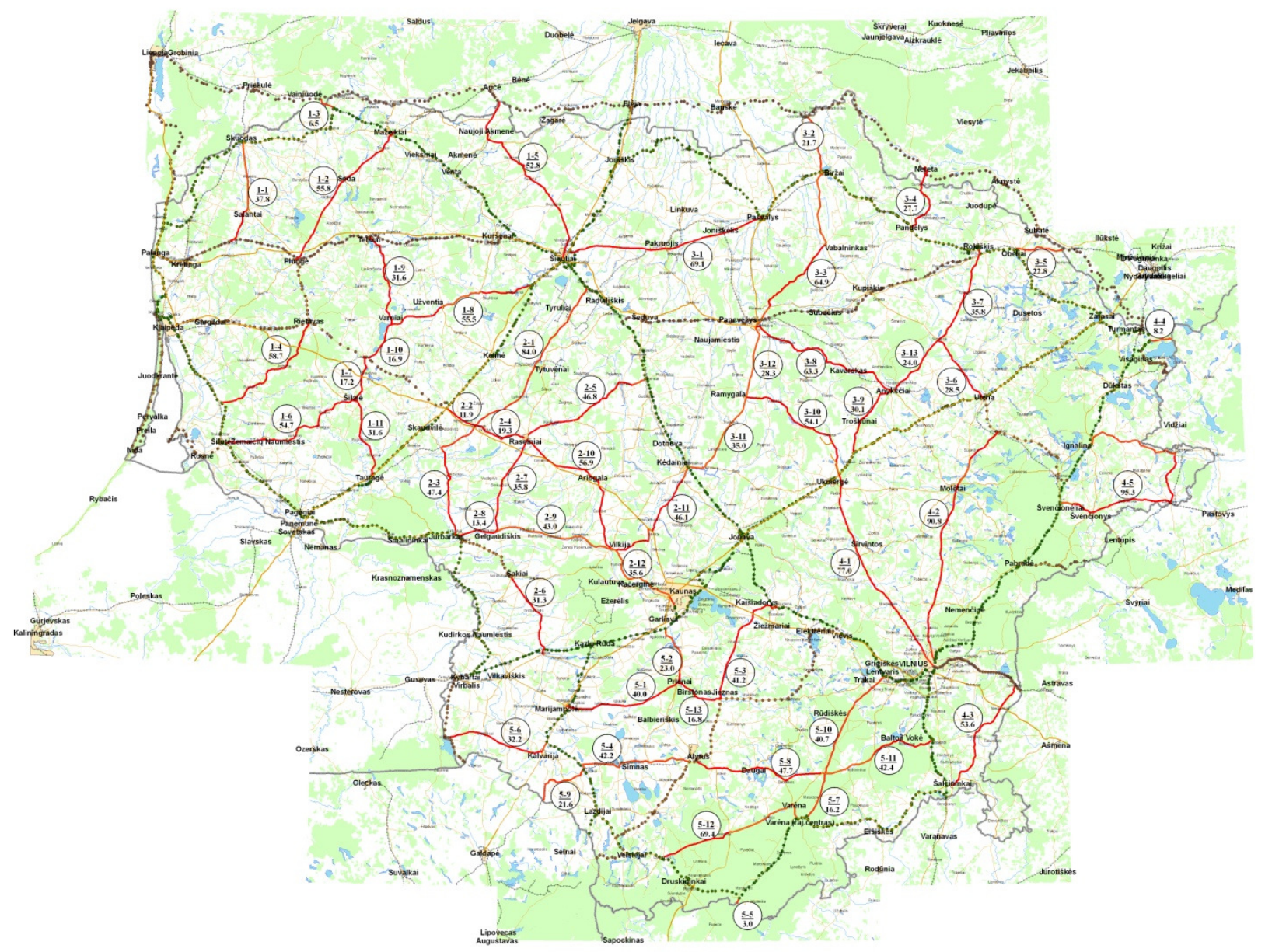

Fig. 2. Scheme of First Order Geodetic Vertical Network of Lithuania (lines in red colour, the line codes and projected lengths are presented in circles)

\section{First experimental results}

The first levelling works were executed in the period from 2007 till 2013. For example, lines of the centre region are shown in Fig. 3.

The two level's sets Trimble DiNi12 were used. They were researched, calibration of coded staffs was done, additional equipment was checked preparing to field expeditions. The field expeditions were organized to fulfil works of the precise levelling. In each expedition 6 employees of Institute of Geodesy ( 2 teams) were formed (levelling teams and preparing the levelling line team). Preparations of levelling line were done by additional levelling instrument N3K and steal cable - to keep distances equal. 35-50 cm length metal stakes were driven into soil or $5 \mathrm{~cm}$ length steal nails with spherical heads in case of asphalt cover.

Digital level DiNi12 and coded staffs LD13 guarantee $0.4 \mathrm{~mm}$ RMS error of double levelling run of one kilometre between reference points. Height differences between points of vertical network were measured forward and backward. Number of stations between neighbouring benchmarks was always even. Staffs were interchanged when direction of levelling was changed. Levelling instrument was levelled in every station when telescope was pointing to the first staff. For the mean reading from the staff about 5 readings with the accuracy of $0.01 \mathrm{~mm}$ were done. Height difference at station measured twice did not exceed $0.25 \mathrm{~mm}$. Distances to the staffs were measured in every station with accuracy of $0.01 \mathrm{~m}$. Length of sight did not exceed $50 \mathrm{~m}$. Difference of sight length in the station was less than $0.5 \mathrm{~m}$. Accumulation of such differences between neighbouring points was below $1.0 \mathrm{~m}$.

Digital levels DiNi12 have opportunity to choose sequence of observations at station. Alternative program was used: in uneven stations - back, forward, forward, back (BFFB); at second station every even station of forward run and in odd stations on back levelling run - forward, back, back, forward (FBBF). Levelling in each station was started from first staff. To start forward levelling second staff was used (levelling staffs was changed). Because of that height differences of the same sign as on stations of forward levelling run were obtained on stations of backward levelling run. For measurements control field book was filled. Normal height of last benchmark, sums of distances between backward (DB) and forward (DF) levelling staffs was deducted after measurements in last station. Length of levelling run was calculated $\mathrm{L}=\mathrm{DB}+\mathrm{DF}$. Computed discrepancy of levelling line height differences $d=\sum h t+\sum$ hd were compared with allowable ones. Temperature of invar bands was measured by contact electronic thermometers Ama-digit ad 30th with $0.1{ }^{\circ} \mathrm{C}$ accuracy. Staff invar band temperature was determined at the height $1.5 \mathrm{~m}$. 


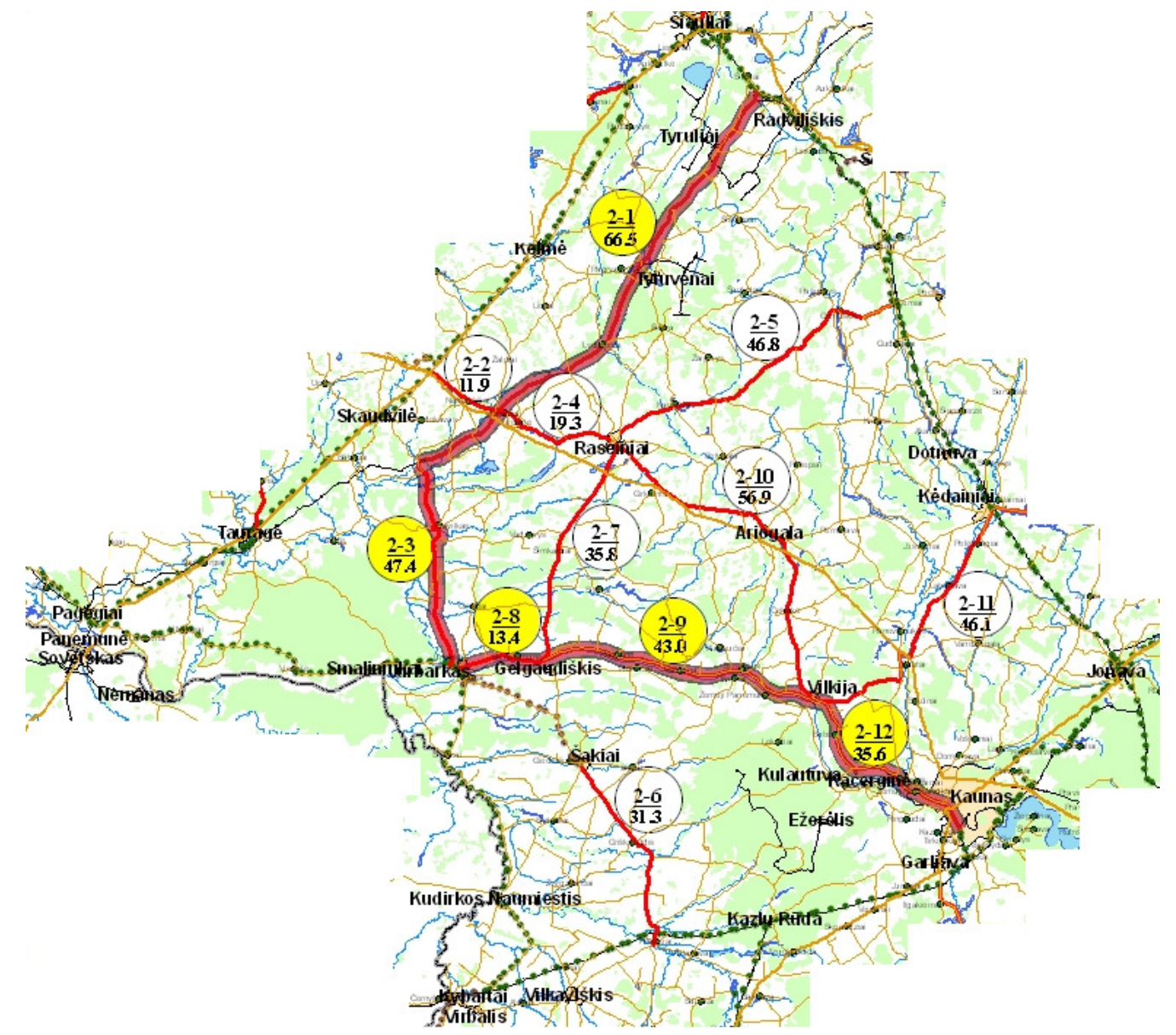

Fig. 3. Levelling lines of Second Order Geodetic Vertical Network in the Centre Region of Lithuania (line No. 2 (red bold) observed in 2013)

The ground benchmarks of vertical network were positioned by 7 GPS receivers Trimble 5700 with Trimble Zephyr Geodetic antennas. LitPOS stations served as fiducial points. Inclined antenna height from three antenna sides was measured in meters and in inches for control. Data registration interval was 10 seconds and cut off angle was $5^{\circ}$. Depending on observation conditions and distances from the GPS stations, measurement sessions lasted for 2.5-4 hours. Baselines connecting National Geodetic Vertical and GPS networks were computed and adjusted by Trimble Total Control software package. Wall benchmarks were coordinated by GPS receiver Trimble 5800 from permanent GPS stations network. Measurements were performed using LitPOS VRS service [13]. Typical scheme of GPS measurements is presented in Fig. 4.

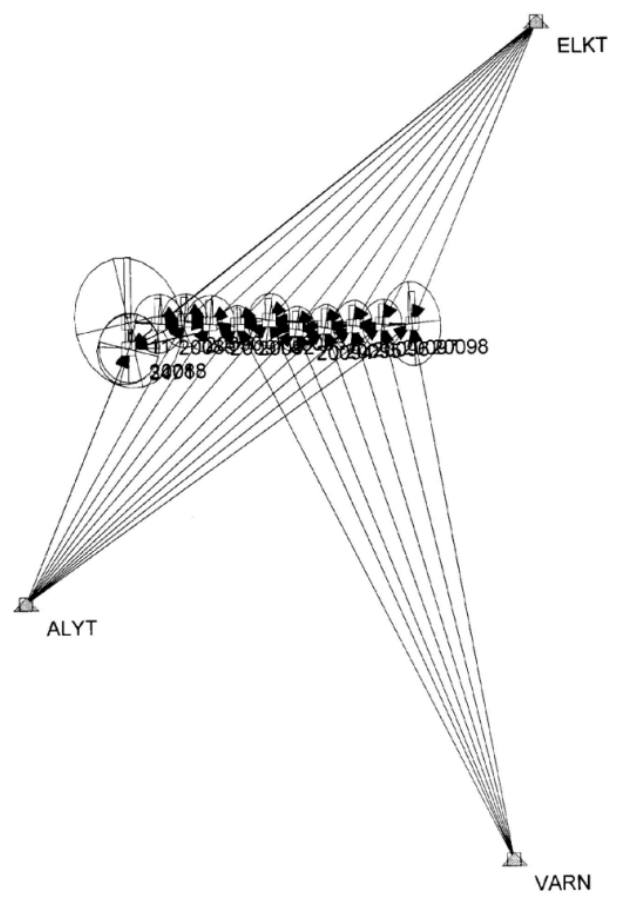

Fig. 4. Typical scheme of ground benchmarks positioning 
The measurements of the gravity acceleration in each network point were performed by Scintrex CG-5 gravimeters. Gravimetric observations were tied with Lithuanian National Gravimetric Network points [14-16]. The example of gravimetric calculations is given below.

At the first step the analysis of daily gravimetric observations should be done [17, 18]. For example, the gravimetric observations were done by two Scintrex CG-5 gravimeters No.182 and No.185. Total number of readings at each point (total number of points in line is 15 ) is 10 by each gravimeter. The maximal differences $\mathrm{d} 1 \mathrm{max}, \mathrm{d} 2 \mathrm{max}$ of the readings and the differences $\mathrm{d} 1 \mathrm{fl}, \mathrm{d} 2 \mathrm{fl}$ of the first and the last reading should be calculated. At this moment the Moon and Sun corrections are not taken into account (Table 1).

Table 1. Differences of readings, mGal

\begin{tabular}{llrlr}
\hline Nr. & d1max & d1fl & d2max & d2fl \\
\hline 1 & 16 & -12 & 17 & -17 \\
2 & 9 & -9 & 7 & -4 \\
3 & 5 & -4 & 22 & -4 \\
4 & 3 & 2 & 8 & -3 \\
5 & 4 & -1 & 18 & -18 \\
6 & 5 & -1 & 9 & 2 \\
7 & 9 & -6 & 6 & -6 \\
8 & 4 & -1 & 25 & 1 \\
9 & 26 & 10 & 12 & -9 \\
10 & 13 & -12 & 12 & -12 \\
11 & 9 & -6 & 10 & -8 \\
12 & 98 & 0 & 73 & -10 \\
13 & 3 & -2 & 14 & -14 \\
14 & 9 & -9 & 17 & -16 \\
15 & 26 & -26 & 15 & -6 \\
\hline
\end{tabular}

At the second step the correted readings due Moon and Sun are calculated and plotted (Fig. 5 and 6).

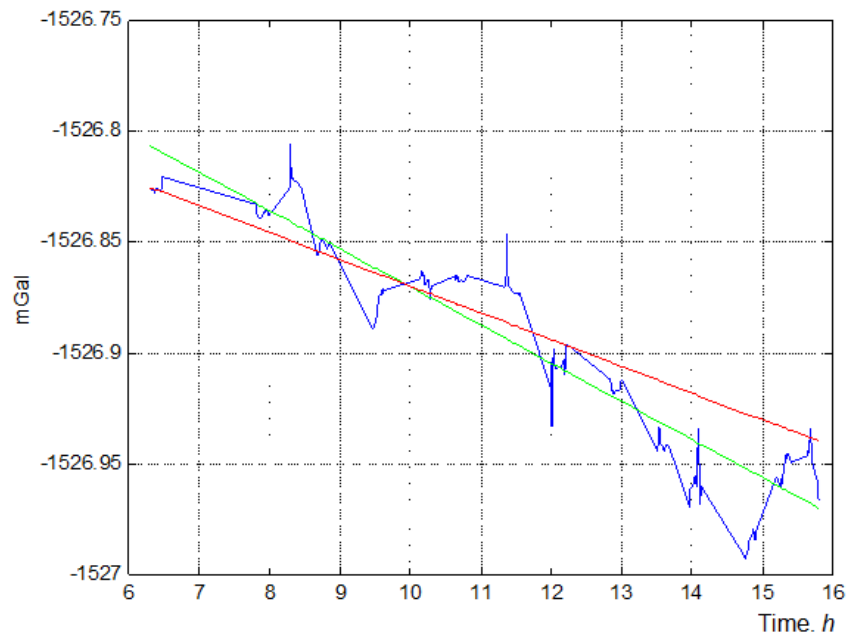

Fig. 5. Differences of corrected readings along a time

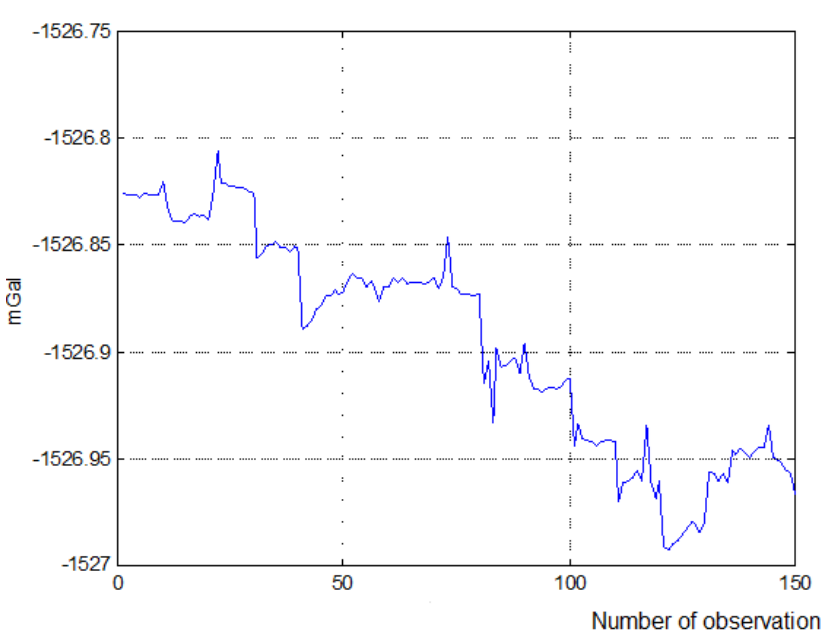

Fig. 6. Differences of corrected readings along a sequence of the observations

At the third step the values of gravity acceleration g2 and g5 and their estimations sd2 and sd5 are calculated. The differences (g2-g5) in $\mu \mathrm{Gal}$ are calculated too, as well as average values of the gravity acceleration gv and its standard deviations mxv. Finally the average values of the gravity acceleration g25 observed by both gravimeters at the gravimeter heights $H_{g}$ and their standard deviations sd25 are derived (Table 2 and Fig. 7). 
Table 2. Gravity acceleration at points in levelling line, mGal

\begin{tabular}{|c|c|c|c|c|c|c|c|c|c|}
\hline $\begin{array}{l}\text { Point } \\
\text { No. }\end{array}$ & g2 & $\mathrm{sd} 2$ & g5 & sd5 & $\begin{array}{l}\text { g2-g5, } \\
\mu \mathrm{Gal}\end{array}$ & gv & g25 & $\mathrm{sd} 25$ & $\begin{array}{l}\text { gv-g25, } \\
\mu \mathrm{Gal}\end{array}$ \\
\hline 1 & 981475.607 & 0.003 & 981475.587 & 0.003 & 20 & 981475.597 & 981475.597 & 0.004 & 0 \\
\hline 3 & 981465.937 & 0.003 & 981465.926 & 0.003 & 11 & 981465.932 & 981465.932 & 0.003 & 0 \\
\hline 1357 & 981489.384 & 0.003 & 981489.376 & 0.004 & 8 & 981489.380 & 981489.380 & 0.004 & 0 \\
\hline 1605 & 981469.256 & 0.003 & 981469.273 & 0.003 & -17 & 981469.264 & 981469.264 & 0.003 & 0 \\
\hline 1901 & 981470.742 & 0.003 & 981470.766 & 0.003 & -24 & 981470.754 & 981470.754 & 0.003 & 0 \\
\hline 8853 & 981474.920 & 0.003 & 981474.914 & 0.003 & 6 & 981474.917 & 981474.917 & 0.003 & 0 \\
\hline 20052 & 981466.046 & 0.003 & 981466.053 & 0.003 & -7 & 981466.050 & 981466.049 & 0.004 & 1 \\
\hline 20054 & 981460.666 & 0.003 & 981460.676 & 0.003 & -10 & 981460.671 & 981460.671 & 0.004 & 0 \\
\hline 25110 & 981463.347 & 0.003 & 981463.378 & 0.003 & -31 & 981463.362 & 981463.362 & 0.004 & 0 \\
\hline 25111 & 981470.582 & 0.003 & 981470.591 & 0.003 & -9 & 981470.586 & 981470.586 & 0.003 & 0 \\
\hline 25113 & 981471.554 & 0.003 & 981471.550 & 0.003 & 4 & 981471.552 & 981471.552 & 0.004 & 0 \\
\hline 25114 & 981476.411 & 0.003 & 981476.376 & 0.003 & 35 & 981476.394 & 981476.394 & 0.004 & 0 \\
\hline 25115 & 981483.032 & 0.003 & 981482.984 & 0.004 & 48 & 981483.008 & 981483.008 & 0.004 & 0 \\
\hline
\end{tabular}

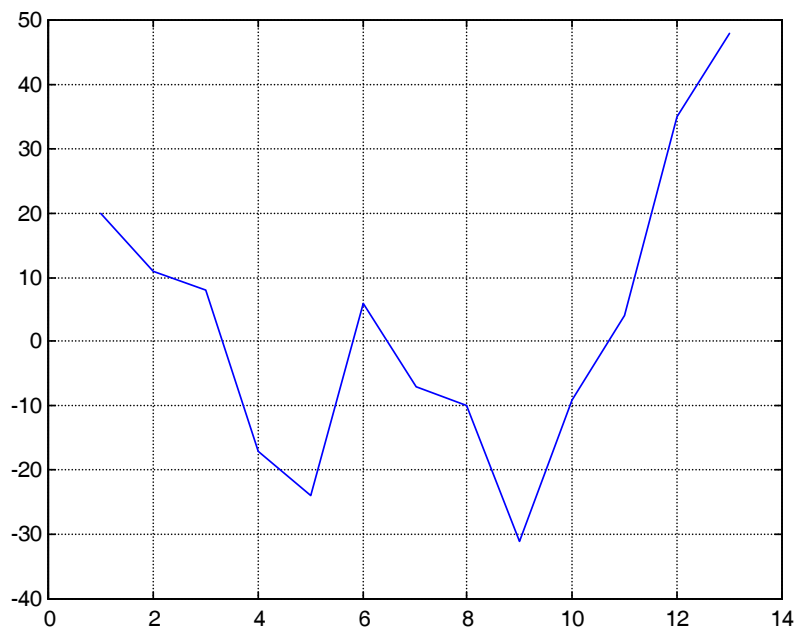

Fig. 7. Differences of gravity acceleration (g2-g5), $\mu \mathrm{Gal}$

At the fourth step the final calculations could be done [19-22]. The gravity acceleration $g_{z}$ at Earth's surface height $H_{z}$ can be obtained by using the formula

$$
g=g_{z}+d g
$$

If $H_{z}>H$, than

$$
d g=\Delta \gamma_{80}(h)-2 \Delta g_{\delta}(h),
$$

here $h=H_{z}-H ; H$ - height of gravimetric point, height correction $\Delta \gamma_{80}(h)=0.3086 h$ was calculated applying the average normal gravity acceleration vertical gradient; correction $\Delta g_{\delta}(h)=2 \pi G \delta h$; gravitational constant $G=6.67259 \cdot 10^{-11} \mathrm{~m}^{3} \mathrm{~kg}^{-1} \mathrm{~s}^{-2}$; density of the Earth's crust $\delta=2.67 \mathrm{~g} \cdot \mathrm{cm}^{-3}$.

If $H_{z}<H$, than

$$
d g=-\Delta \gamma_{80}(h)
$$

here $h=H-H_{z}$.

If gravity acceleration was measured above Earth's surface, than its value at the Earth's surface will be

$$
g_{z}=g+\Delta \gamma_{80}(h) \text {. }
$$

The Bouguer dgB80 and free air dglo anomalies at the height $H_{z}$ are calculated also (Table 3 and Fig. 8). 
Table 3. Gravity acceleration and anomalies

\begin{tabular}{|c|c|c|c|c|c|c|c|c|c|c|}
\hline No. & Point code & $\begin{array}{l}H \\
\mathrm{~m}\end{array}$ & $\begin{array}{l}B \\
\text { o }\end{array}$ & $\begin{array}{l}H_{g} \\
\mathrm{~m}\end{array}$ & $\begin{array}{l}H_{z} \\
\mathrm{~m}\end{array}$ & $\begin{array}{l}\text { g25, } \\
\text { mGal }\end{array}$ & $\begin{array}{l}\mathrm{gz}, \\
\mathrm{mGal}\end{array}$ & $\begin{array}{l}\text { dglo, } \\
\text { mGal }\end{array}$ & $\begin{array}{l}\mathrm{dgB} 80, \\
\text { mGal }\end{array}$ & $\begin{array}{l}\mathrm{g}, \\
\mathrm{mGal}\end{array}$ \\
\hline 1 & $83 \mathrm{~V}-1357$ & 130.245 & 54.98506126 & 131.665 & 131.75 & 981489.380 & 981489.373 & 24.709 & 9.961 & 981489.500 \\
\hline 2 & $83 \mathrm{~V} 25115$ & 143.251 & 54.98034536 & 144.541 & 144.56 & 981483.008 & 981483.006 & 22.694 & 6.512 & 981483.117 \\
\hline 3 & $83 V-5958$ & 147.487 & 54.97750000 & 148.091 & 148.09 & 981480.050 & 981480.050 & 21.069 & 4.492 & 981480.101 \\
\hline 4 & $83 \mathrm{~V} 25114$ & 154.090 & 54.98166667 & 155.730 & 155.74 & 981476.394 & 981476.393 & 19.416 & 1.982 & 981476.532 \\
\hline 5 & 83V----1 & 142.370 & 54.98508510 & 142.110 & 142.11 & 981475.597 & 981475.597 & 14.126 & -1.782 & 981475.517 \\
\hline 6 & $83 \mathrm{~V}-8853$ & 148.052 & 54.97880556 & 148.582 & 148.64 & 981474.917 & 981474.912 & 15.989 & -0.649 & 981474.962 \\
\hline 7 & $83 V 25113$ & 149.482 & 54.97447271 & 149.552 & 149.56 & 981471.552 & 981471.551 & 13.281 & -3.460 & 981471.558 \\
\hline 8 & 83S-1901 & 150.033 & 54.97576841 & 150.513 & 150.57 & 981470.754 & 981470.749 & 12.680 & -4.174 & 981470.794 \\
\hline 9 & $83 V 25111$ & 146.749 & 54.97351389 & 146.969 & 146.97 & 981470.586 & 981470.586 & 11.599 & -4.853 & 981470.605 \\
\hline 10 & $83 \mathrm{~V}-1605$ & 147.234 & 54.96977778 & 147.574 & 147.61 & 981469.264 & 981469.261 & 10.790 & -5.734 & 981469.293 \\
\hline 11 & $83 \mathrm{~V}-0003$ & 153.135 & 54.96330556 & 153.135 & 153.14 & 981465.932 & 981465.932 & 9.717 & -7.425 & 981465.932 \\
\hline 12 & $83 \mathrm{~V} 20052$ & 153.148 & 54.97879936 & 153.218 & 153.30 & 981466.049 & 981466.042 & 8.557 & -8.604 & 981466.055 \\
\hline 13 & $83 \mathrm{~V}-6061$ & 158.779 & 54.98444444 & 159.781 & 159.78 & 981465.461 & 981465.461 & 9.493 & -8.393 & 981465.546 \\
\hline 14 & $83 V 25110$ & 162.257 & 54.98445000 & 161.697 & 161.74 & 981463.362 & 981463.358 & 7.994 & -10.111 & 981463.199 \\
\hline 15 & $83 \mathrm{~V} 20054$ & 169.021 & 54.98768836 & 169.091 & 169.17 & 981460.671 & 981460.664 & 7.315 & -11.622 & 981460.677 \\
\hline
\end{tabular}

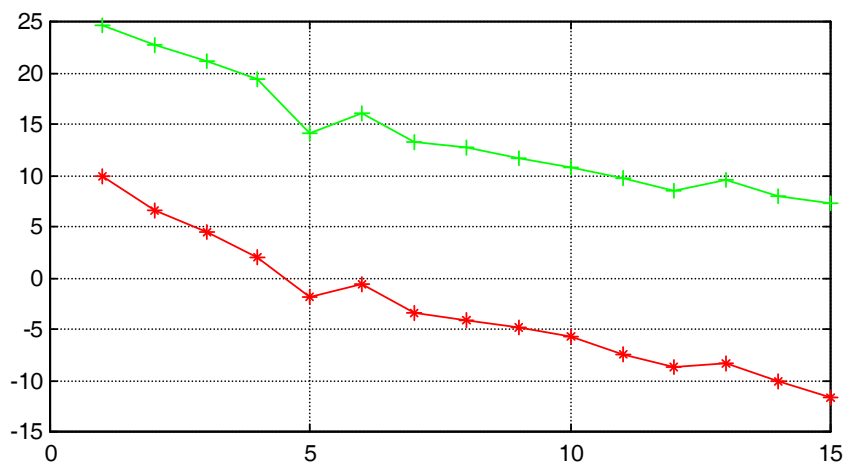

Fig. 8. Changes of gravity anomalies along the levelling line, mGal (dglo - - - +-, dgB80 - - *-.

\section{Quality evaluation of the precise levelling}

Quality of the precise levelling of the Geodetic Vertical Second Order Network is controlled according to height difference tolerances computed for the levelling run. All discrepancies of height difference mentioned above should do not exceed:

$$
d=3.0 \mathrm{~mm} \sqrt{L},
$$

where $L$ - length of levelling line in $\mathrm{km}$.

After finishing the precise levelling of few levelling lines based on double (forward and backward) run levelling discrepancies distributions should be estimated. Levelling results are correct if they satisfy formula (5). In the period from 2006 till 2013 the length of the precise levelling lines is $908 \mathrm{~km}$ (total 705 runs). The discrepancies of formulae (5) were distributed into intervals from 0 increasing every $\pm 0.5 \mathrm{~mm}:|0-0.5| \mathrm{mm} \sqrt{L},|0.5-1.0| \mathrm{mm} \sqrt{L},|1,0-1,5| \mathrm{mm} \sqrt{L}$, $|1.5-2.0| \mathrm{mm} \sqrt{L}$ and $\geq|2.0| \mathrm{mm} \sqrt{L}$. Calculations are presented in Table 4 and Figure 9.

Levelling run weight unit (one kilometre) RMS error was computed fromwell known formula

$$
\mu=\sqrt{\frac{\left[p d^{2}\right]}{2 n}},
$$

where $p=1 / L$-weight of height difference, $L$ - distance between benchmarks in kilometres, $d$ - double run levelling height differences discrepancy, $n-$ number of discrepancies. 
Table 4. Precise levelling quality evaluation basing on double run levelling discrepancies

\begin{tabular}{|c|c|c|c|c|c|c|}
\hline \multirow{2}{*}{ Levelling lines } & \multirow{2}{*}{$\begin{array}{l}\begin{array}{l}\text { Number of } \\
\text { levelling runs }\end{array} \\
\text { Distances, } \\
\mathrm{km}\end{array}$} & \multirow{2}{*}{$|0-0.5| \mathrm{mm} \sqrt{L}$} & \multirow{2}{*}{$|0.5-1.0| \mathrm{mm} \sqrt{L}$} & \multirow{2}{*}{$|1.0-1.5| \mathrm{mm} \sqrt{L}$} & \multirow{2}{*}{$|1.5-2.0| \mathrm{mm} \sqrt{L}$} & \multirow{2}{*}{$\geq|2.0| \mathrm{mm} \sqrt{L}$} \\
\hline & & & & & & \\
\hline \multirow{2}{*}{$\begin{array}{l}\text { Palanga - } \\
\text { Kretinga }\end{array}$} & 12 & 6 & 5 & 1 & - & - \\
\hline & 13.15 & 6.06 & 6.47 & 0.62 & - & - \\
\hline \multirow{2}{*}{$\begin{array}{l}\text { Kužiai - } \\
\text { Telšiai }\end{array}$} & 45 & 25 & 13 & 4 & 3 & - \\
\hline & 58.12 & 31.70 & 18.43 & 5.09 & 2.90 & - \\
\hline \multirow{2}{*}{$\begin{array}{l}\text { Šeduva-- } \\
\text { Panevėžys }\end{array}$} & 33 & 19 & 7 & 6 & 1 & - \\
\hline & 48.91 & 25.49 & 12.93 & 9.97 & 0.52 & - \\
\hline \multirow{2}{*}{$\begin{array}{l}\text { Kiduliai - } \\
\text { Šakiai }\end{array}$} & 21 & 15 & 2 & 4 & - & - \\
\hline & 24.86 & 18.35 & 2.51 & 4.00 & - & - \\
\hline \multirow{2}{*}{$\begin{array}{l}\text { Klaipèda - } \\
\text { Rietavas }\end{array}$} & 37 & 16 & 13 & 6 & 2 & - \\
\hline & 52.54 & 23.48 & 17.40 & 7.23 & 4.43 & - \\
\hline \multirow{2}{*}{$\begin{array}{l}\text { Gudeliai - } \\
\text { Alytus }\end{array}$} & 32 & 17 & 9 & 5 & 1 & - \\
\hline & 44.03 & 23.02 & 11.59 & 8.92 & 0.50 & - \\
\hline \multirow{2}{*}{$\begin{array}{l}\text { Vievis - } \\
\text { Elektrènai }\end{array}$} & 10 & 7 & 2 & 1 & - & - \\
\hline & 12.02 & 7.62 & 3.02 & 1.38 & - & - \\
\hline \multirow{2}{*}{$\begin{array}{l}\text { Rokiškis - } \\
\text { Panevėžys }\end{array}$} & 82 & 40 & 24 & 12 & 4 & 2 \\
\hline & 84.43 & 43.42 & 23.40 & 11.22 & 4.15 & 2.24 \\
\hline \multirow{2}{*}{$\begin{array}{l}\text { Telšiai - } \\
\text { Plungė - } \\
\text { Kretinga }\end{array}$} & 98 & 47 & 37 & 12 & 2 & - \\
\hline & 74.28 & 35.25 & 29.02 & 8.01 & 2.00 & - \\
\hline \multirow{2}{*}{$\begin{array}{l}\text { Šilutè - Nida - } \\
\text { Klaipeda }\end{array}$} & 68 & 36 & 22 & 7 & 2 & 1 \\
\hline & 102.80 & 46.02 & 40.65 & 11.18 & 1.94 & 3.01 \\
\hline \multirow{2}{*}{$\begin{array}{l}\text { Alytus - } \\
\text { Elektrènai }\end{array}$} & 48 & 25 & 12 & 10 & 1 & - \\
\hline & 80.57 & 41.43 & 19.94 & 17.51 & 1.69 & - \\
\hline \multirow{2}{*}{$\begin{array}{l}\text { Vilnius - } \\
\text { Šumskas }\end{array}$} & 43 & 24 & 11 & 6 & 1 & 1 \\
\hline & 36.37 & 21.00 & 9.62 & 4.67 & 0.13 & 0.95 \\
\hline \multirow{2}{*}{$\begin{array}{l}\text { Pabradè - } \\
\text { Gelednè }\end{array}$} & 14 & 7 & 2 & 4 & 1 & - \\
\hline & 25.17 & 11.95 & 4.59 & 7.51 & 1.32 & - \\
\hline \multirow{2}{*}{$\begin{array}{l}\text { Utena - } \\
\text { Švenčionèliai }\end{array}$} & 23 & 9 & 12 & 2 & - & - \\
\hline & 49.18 & 18.68 & 26.98 & 3.52 & - & - \\
\hline \multirow{2}{*}{$\begin{array}{l}\text { Visaginas - } \\
\text { Varniškiai }\end{array}$} & 22 & 10 & 3 & 7 & 2 & - \\
\hline & 21.70 & 7.74 & 3.00 & 7.63 & 3.33 & - \\
\hline \multirow{2}{*}{$\begin{array}{l}\text { Plungè - } \\
\text { Rietavas - } \\
\text { Stulgiai }\end{array}$} & 47 & 26 & 12 & 5 & 3 & 1 \\
\hline & 85.83 & 48.63 & 20.45 & 7.86 & 6.66 & 2.23 \\
\hline \multirow{2}{*}{$\begin{array}{l}\text { Kaunas - } \\
\text { Vilkija }\end{array}$} & 26 & 15 & 9 & - & 2 & - \\
\hline & 34.26 & 19.81 & 12.47 & - & 1.98 & - \\
\hline \multirow[t]{2}{*}{ Vilkija - Šilinè } & 32 & 17 & 11 & 3 & 1 & - \\
\hline & 43.20 & 24.31 & 13.01 & 4.81 & 1.07 & - \\
\hline Šilinè - & 12 & 10 & 1 & 1 & - & - \\
\hline Jurbarkas & 16.40 & 13.57 & 1.01 & 1.82 & - & - \\
\hline$\Gamma$ & $705(100 \%)$ & $371(52.6 \%)$ & $207(29.4 \%)$ & $96(3.6 \%)$ & $26(3.7 \%)$ & $5(0.7 \%)$ \\
\hline 2 & $908(100 \%)$ & $467(51.5 \%)$ & $276(30.5 \%)$ & $122(13.5 \%)$ & $32(3.6 \%)$ & $8(0.9 \%)$ \\
\hline
\end{tabular}

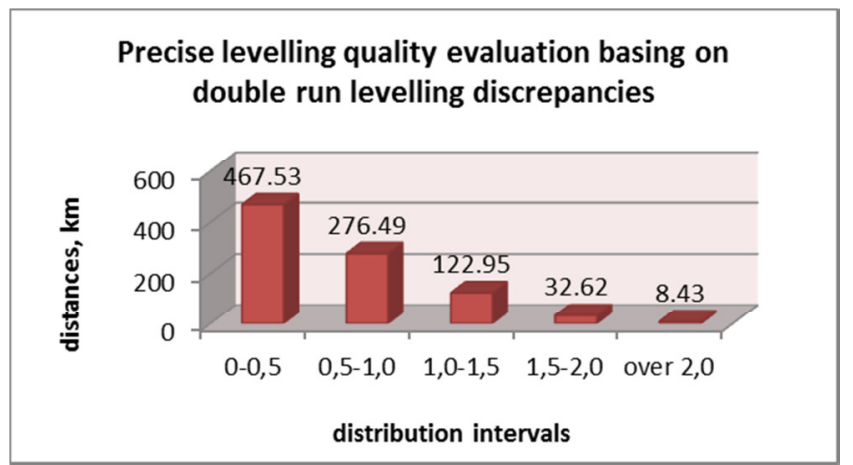

Fig. 9. Results of quality evaluation of precise levelling 
Double run levelling one km length RMS error was computed:

$$
m_{k m}=\frac{\mu}{\sqrt{2}} .
$$

The accuracy characteristics of every levelling line are presented in Table 5.

Table 5. The accuracy characteristics

\begin{tabular}{|c|c|c|}
\hline No. & Levelling line & $m_{k m}, \mathrm{~mm}$ \\
\hline 1 & Palanga - Kretinga & 0.31 \\
\hline 2 & Kužiai - Telšiai & 0.32 \\
\hline 3 & Šeduva - Panevėžys & 0.35 \\
\hline 4 & Kiduliai - Šakiai & 0.31 \\
\hline 5 & Klaipèda-Rietavas & 0.46 \\
\hline 6 & Gudeliai - Alytus & 0.35 \\
\hline 7 & Vievis - Elektrenai & 0.25 \\
\hline 8 & Šilutè - Nida - Klaipèda & 0.12 \\
\hline 9 & Rokiškis - Panevėžys & 0.38 \\
\hline 10 & Telšiai - Plungè - Kretinga & 0.36 \\
\hline 11 & Alytus - Elektrènai & 0.37 \\
\hline 12 & Vilnius - Šumskas & 0.36 \\
\hline 13 & Pabradè - Gelednè & 0.43 \\
\hline 14 & Utena - Švenčionèliai & 0.35 \\
\hline 15 & Visaginas - Varniškiai & 0.47 \\
\hline 16 & Plungè - Rietavas - Stulgiai & 0.37 \\
\hline 17 & Kaunas - Vilkija & 0.12 \\
\hline 18 & Vilkija-ŠSilinè & 0.35 \\
\hline 19 & Šilinè - Jurbarkas & 0.47 \\
\hline
\end{tabular}

Based on Table 5 data it is clear that the double run one kilometre levelling RMS error does not exceeds $0.5 \mathrm{~mm}$. We could conclude that the levellings carried out so far is characterized by high precision.

\section{Conclusions}

1. The project of National Second Order Geodetic Vertical Network was prepared. The network is not so dense as it was initially designed. The network is composed of 70 lines, which total length is about $3000 \mathrm{~km}$. Old levelling lines are selected for most of the projected lines. About 2250 benchmarks, 1200 of them newly built should be included into the Second Order Network.

2. Lithuanian digital geoid model will be improved after completing the First and Second Order of Geodetic Vertical Network. This will enable to derive normal height from ellipsoidal heights determined by GPS and replace traditional levelling of lower orders.

\section{Acknowledgements}

The research was supported by a grant No. 1DPS-(4.27)-1675/10552 of the National Land Service of Lithuania.

\section{References}

[1] Petroškevičius, P.; Paršeliūnas, E. 1998. European Vertical Network expansion to Lithuania. Geodesy and Cartography (Geodezija ir kartografija) 24(3): 133-145. ISSN 1392-1541 (in Lithuanian).

[2] Petroskevicius, P.; Zakarevicius, A.; Parseliunas, E. 2005. Premises for Lithuanian gravity and height systems adoption, in The $6^{\text {th }}$ International Conference „Environmental Engineering“: Selected papers (26-27 May 2005, Vilnius, Lithuania) 2: 985-992. ISBN 9986-05-851-1.

[3] Petroskevicius, P.; et al. 2005. Analysis Of Normal Height Differences Determination In Lithuanian National Geodetic Vertical Network, in The 6th International Conference „Environmental Engineering “: Selected papers (26-27 May 2005, Vilnius, Lithuania) 2: 975-984. ISBN 9986-05-851-1.

[4] Būga, A.; Kolosovskis, R.; Obuchovski, R.; Paršeliūnas, E.; Petroškevičius, P.; Šleiteris, E.; Urbanas, S.; Zakarevicius, A. 2006. Ongoing Developments of the Lithuanian National Geodetic Control, Mitteilungen des Bundesamtes für Kartographie und Geodäsie 38: 255-262. ISSN 14363445 . 
[5] Petroskevicius, P.; Putrimas, R.; Krikstaponis, B.; Buga, A.; Neseckas, A.; Obuchovski, R.; Stepanoviene, J.; Tumeliene, E.; Viskontas, P.; Zigmantiene, E. 2005. Analysis of normal height differences determination in Lithuanian national geodetic vertical network, in The $6^{\text {th }}$ International Conference "Environmental Engineering” Selected Papers 2: 975-984.

[6] Putrimas, R.; Krikstaponis, B.; Neseckas, A.; Kolosovskis, R.; Stepanoviene, J.; Tumeliene, E.; Viskontas, P.; Zigmantiene, E. 2008. Results of Lithuanian national geodetic vertical first order network development, in The $7^{\text {th }}$ International Conference "Environmental Engineering", Selected Papers 3: 1446-1456.

[7] Parseliunas, E. 2008. Integration of the Lithuanian vertical network into united European leveling network, in The $7^{\text {th }}$ International Conference “Environmental Engineering”, Selected papers (22-23 May 2008, Vilnius, Lithuania) 3: 1415-1421. ISBN 978-9955-28-265-5.

[8] Krikstaponis, B.; Parseliunas, E.; Petroskevicius, P.; Putrimas, R.; Urbanas, S.; Zakarevicius, A. 2007. Realization of the Vertical Datum and Height System of Lithuania, Harita Dergisi 18: 142-147. ISSN 1300-5790.

[9] Aleksejenko, I.; Sakne, J.; Kalinka, M.; Petroskevicius, P.; Parseliunas, E.; Viskontas, P.; Kalantaite, A.; Urbanas, S. 2011. Levelling network connection between Latvia and Lithuania, in The $8^{\text {th }}$ International Conference "Environmental Engineering” Selected Papers 4. Vilnius Gediminas Technical University Press "Technika", 2011.

[10] Aleksejenko, I.; Sakne, J.; Kalinka, M.; Reiniks, M.; Kalantaitė, A.; Krikštaponis, B.; Paršeliūnas, E.; Petroškevičius, P.; Viskontas, P. 2012. The united geodetic vertical network of Latvia and Lithuania, Geodesy and cartography 38(1): 9-19. ISSN 2029-6991.

[11] Lyszkowicz, A. ; Bernatowicz, A. 2011. Statistical Analysis of the Fourth Precise Levelling Campaign in Poland, Techn. Sc. 14(2): 263-278.

[12] Kazakevicius, S. V.; Tumeliene, E. 2005. Project Of Lithuanian National Second Order Geodetic Vertical Network, in The $6^{\text {th }}$ International Conference "Environmental Engineering”, Selected papers (26-27 May 2005, Vilnius, Lithuania) 2: 897-902. ISBN 9986-05-851-1.

[13] Paršeliūnas, E.-K. 2008. LitPOS - a service for precise positioning in real time, in The $25^{\text {th }}$ International Symposium on Automation and Robotics in Construction (ISARC 2008), selested papers, June 26-29, 2008 Vilnius, Lithuania. Vilnius : Technika, 2008, p. 379-383. ISBN 9789955283041.

[14] Sas-Uhrynowski, A.; Mroczek, S.; Sas A.; Petroškevičius, P.; Obuchowski, R.; Rimkus, D. 2002. Establishment of Lithuanian national gravimetric first order network, Geodezija ir kartografija 28(3): 75-82.

[15] Paršeliūnas, E., Petroškevičius, P. 2007. Quality of Lithuanian National Gravimetric Network, Journal of Mapping (18): 388-392.

[16] Paršeliūnas, E.; Obuchovski, R.; Birvydienè, R.; Petroškevičius, P.; Zakarevičius, A.; Aksamitauskas, V. Č.; Rybokas, M. 2010. Some issues of the national gravimetric network development in Lithuania, Journal of vibroengineering 12: 685-690. ISSN 1392-8716.

[17] Paršeliūnas, E.; Petroškevičius, P.; Obuchovski, R.; Birvydienė, R. 2013. An investigation of the automatic relative gravimeters, in Mechatronic Systems and Materials V. Solid State Phenomena. Switzerland : Trans Tech Publications Ltd. (Solid State Phenomena, Vol. 199, ISSN 1392-4044). ISSN 1012-0394. ISBN 9783037856451. 199: 261-266

[18] Petroškevičius, P.; Birvydienè, R.; Obuchovski, R.; Paršeliūnas, E. 2011. Investigation into measurements using gravimeters SCINTREX CG-5, Geodesy and cartography 37(4): 154-158. ISSN 2029-6991.

[19] Petroškevičius, P.; Popovas, D.; Krikštaponis, B.; Putrimas, R.; Būga, A.; Obuchovski, R. 2008. Estimation of gravity field non-homogeneity and variation for the vertical network observations, in The $7^{\text {th }}$ International conference "Environmental engineering", Selected Papers (22-23 May 2008, Vilnius, Lithuania) 3: 1439-1445.

[20] Petroškevičius, P. 2004. Gravitation field effect on geodetic observations. Vilnius: Technika. 290 p. (in Lithuanian).

[21] Petroškevičius, P. 2000. Evaluation of equipotential surfaces non-paralellity, Geodesy and Cartography 26(2): 53-56 (in Lithuanian).

[22] Petroškevičius, P. 2001. Evaluation of celestial bodies influence on vertical, Geodesy and Cartography 27(2): 49-54. ISSN 1392-1541 (in Lithuanian). 